\title{
Evaluation of Angiopoietin 2 Serum Level in Lichen Planus and Psoriasis Patients
}

\author{
Mohamed AbdElkader Teama ${ }^{1}$, Hagar Awad Bessar ${ }^{1}$, \\ Noha Mohamed El-Sayed ${ }^{2}$, Tawhed Mahmoud Ismail Mahmoud ${ }^{1}$ \\ Departments of ${ }^{1}$ Dermatology, Venereology \& Andrology and ${ }^{2}$ Medical Microbiology \& Immunology, \\ Faculty of Medicine - Zagazig University. \\ *Corresponding Author: Tawhed Mahmoud Ismail Mahmoud, Mobile: (+20)01024683929, \\ Email: mosad8rashed@gmail.com
}

\begin{abstract}
Background: Psoriasis and lichen planus (LP) are common chronic inflammatory skin diseases. The exact pathogenesis of these diseases until now is not fully determined. Angiogenesis is tightly regulated by a balance between pro- and anti-angiogenic mediators.

Objective: To assess the relation of Ang-2 serum level with angiogenesis and inflammation that occurs in psoriasis and lichen planus.

Patients and Methods: Thirty adult patients were included in this study. They were divided into 2 groups, 15 patients each, of psoriasis and lichen planus ( 8 patients with combined cutaneous and oral LP and 7 patients with cutaneous LP only). In addition to fifteen healthy subjects taken as control. The activity of the diseases was estimated at the time of examination using specific activity index for each disease. The serum level of angiopoietin 2 had been estimated by ELISA.

Results: It was found that these diseases had significantly higher serum levels of angiopoietin 2 than the control group. The serum levels of angiopoietin 2 were higher in psoriasis compared to lichen planus, but the difference between psoriasis and lichen planus was not statistically significant. There was significant positive correlation between serum levels of angiopoietin 2 and the clinical severity of psoriasis. On the other hand, there was no correlation between serum level of angiopoietin 2 and the clinical severity of lichen planus.

Conclusion: An important evidence on the importance of angiopoietin 2 serum level in pathogenesis of lichen planus as well as psoriasis. It elevated in serum of psoriasis and lichen planus patients that reflects its role in angiogenesis and inflammation that are important events in the development of these diseases.
\end{abstract}

Keywords: Angiopoietin 2, Lichen planus, Psoriasis, Evaluation.

\section{INTRODUCTION}

Psoriasis and lichen planus are chronic relapsing inflammatory skin diseases. They may lead to marked disability affecting the physical and emotional wellbeing of patients. Both combined cost of long-term therapy and social burden of this disease have a major impact not only on the patient, but also on health care systems. Recent studies indicate that severe and long-standing psoriasis is associated with co-morbidities, as well as a shorter life span due to cardiovascular mortality ${ }^{(\mathbf{1})}$.

These diseases are multifactorial, with complex interactions of innate and adaptive immune responses based on a strong genetic predisposition and triggered by environmental factors. The exact pathogenesis of these diseases now is not fully determined ${ }^{(2)}$.

In addition, recent studies suggest that angiogenesis is essential for the triggering of psoriatic pathology beside being the key mechanism in psoriasis by marked increase in the microvasculature and underlying marker of lichen planus activity ${ }^{(3)}$. So, the critical role of angiogenesis in psoriasis and lichen planus pathogenesis is strongly suggested. In angiogenesis, the prime angiogenic factor is VEGF, which has been characterized to have the role in proliferation, survival and cell migration, as well as vascular permeability ${ }^{(4)}$.

In addition to the VEGF signaling, studies have drawn much attention to the contribution of aberrantly activated Ang-2 signaling to angiogenesis development. It has been identified to interact with VEGF to determine the fate of blood vessels during angiogenesis as it is very essential for controlling sprouting angiogenesis, vascular remodeling and the transition between the quiescent and the activated status of ECs ${ }^{(\mathbf{5})}$. Beside its role in angiogenesis, Ang2 has a positive association with chronic inflammation and psoriasis. For instance, it induces vascular leakage and sensitizes endothelial cells for signaling molecules like tumor necrosis factor-á (TNF-á). Furthermore, it has paracrine eff ects on leukocytes, specifically myeloid cells, leading to monocytes' migration and up/down regulation of cytokines in neutrophilic granulocytes and macrophages ${ }^{(6)}$.

The study aimed to o detect the role of Angiopoietin 2 in the pathogenesis of psoriasis and lichen planus.

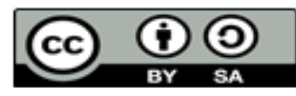

Received:7 /7/2020

Accepted:22 /8 /2020

This article is an open access article distributed under the terms and conditions of the Creative Commons Attribution (CC BY-SA) license (http://creativecommons.org/licenses/by/4.0/) 


\section{PATIENTS AND METHODS}

The study was carried out in the Department of Dermatology and Venereology, Faculty of Medicine, Zagazig University during the period from February 2019 to November 2019. The ELISA test was done at the immunology lab of the Medical Microbiology and Immunology Department, Faculty of Medicine, Zagazig University.

\section{Ethical Considerations}

The protocols and informed consent forms used in this study were approved by the Institutional Review Board (IRB) of Zagazig University (No. of Approval: 5207).

All participants signed a written informed consent and filled a written survey including demographic and clinical data.

Type of Study: Case control study

Sample Selection:

The study included 30 Patients and 15 controls. They were assigned to three groups;

- The first group included 15 patients with psoriasis vulgaris.

- The second group included 15 patients with lichen planus.

- The third group included 15 age and sex matched healthy controls.

Inclusion criteria:

1. Subjects in case group, patients diagnosed clinically

- Lichen planus, on the basis of typical appearance of skin lesions and oral ones.

- Psoriasis, on the basis of typical appearance of skin lesions.

2. Median age (25-59), males and females.

\section{Exclusion criteria}

- Participant refusal.

- Pregnancy.

- Immune deficiencies.

- Patients known to be HIV or otherwise Immunocompromised.

- Patients with graft versus host disease or other autoimmune disease.

- Patients with lichenoid reactions due to contact, drugs or smoking.

- Patients with active medical conditions or malignancies.

\section{A- All patients were subjected to history taking}

1. Detailed personal history.

2. History of present illness,

- Duration of disease, onset, course, extension, treatment used (topical or systemic) and other dermatological problems.

3. Family history.

B- Complete general examination, to exclude any associated other dermatological diseases

C- Complete dermatological examination, to describe the lesions

- Site, number, pattern of distribution, shape and buccal examination for identification of oral lichen planus.

The severity of psoriasis was measured by Psoriasis Area and Severity Index (PASI) according to the method described by Robinson ${ }^{(7)}$ (Fig 1).

\section{PASI calculation:}

A representative area of psoriasis is selected for each body region. The intensity of redness, thickness and scaling of the psoriasis is assessed as none (0), mild (1), moderate (2), severe (3) or very severe (4). 


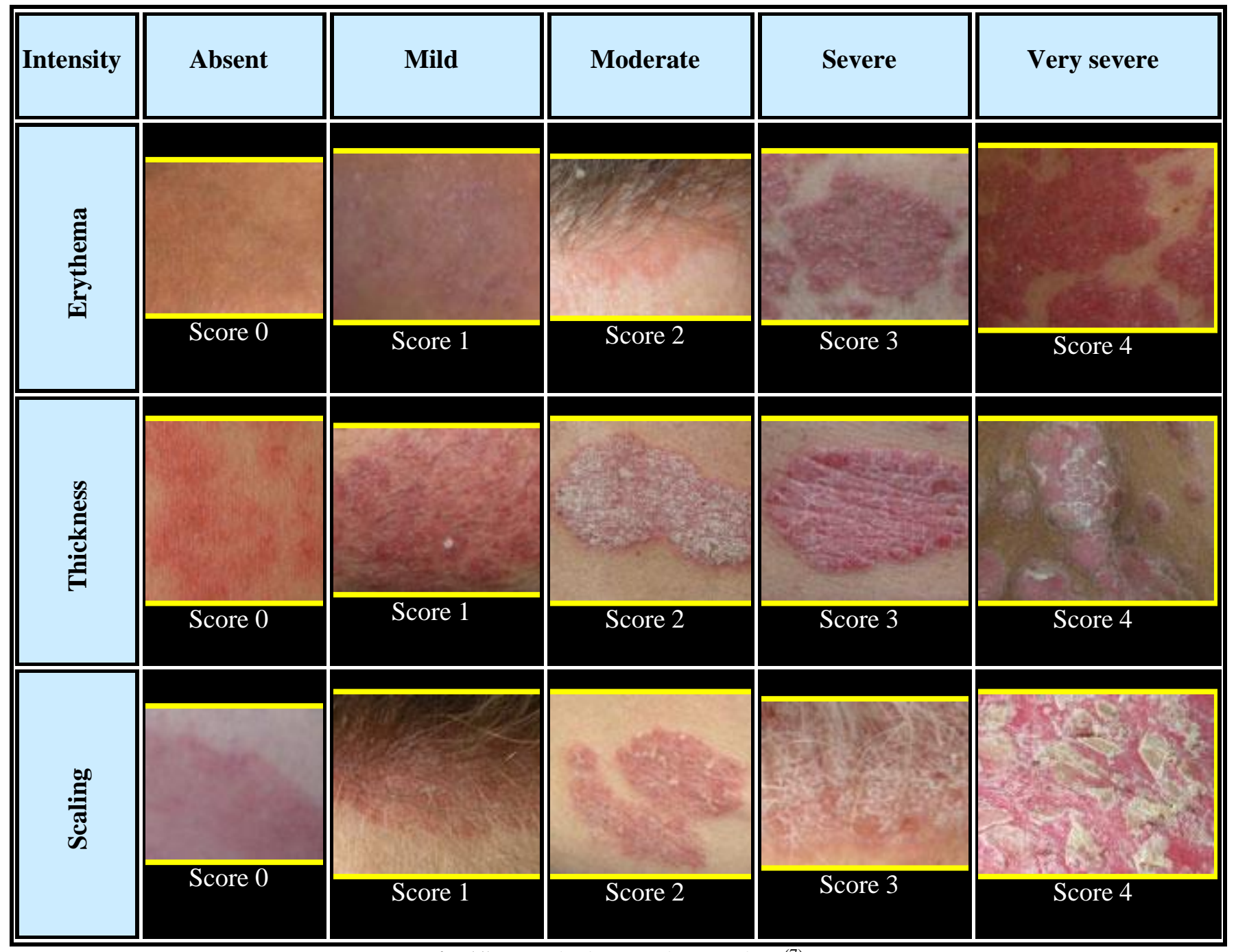

Fig (1): Psoriasis severity scoring ${ }^{(7)}$

\section{Calculation for intensity:}

The three intensity scores were added up for each of the four body regions to give subtotals A1, A2, A3 and A4.

Each subtotal was multiplied by the body surface area represented by that region.

- A1 x 0.1 gives B1

- $\mathrm{A} 2 \times 0.2$ gives $\mathrm{B} 2$

- $\mathrm{A} 3 \times 0.3$ gives $\mathrm{B} 3$

- A4 x 0.4 gives B4

Area

The percentage area affected by psoriasis is evaluated in the four regions of the body. In each region, the area is expressed as nil (0), 1$9 \%(1), 13-29 \%$ (2), 30-49\% (3), 50-69\% (4), $70-89 \%$ (5) or $90-100 \%$ (6).

- Head and neck

- Upper limbs

- Trunk
- Lower limbs

Calculations for area

Each of the body area scores is multiplied by the area affected.

- $\mathrm{B} 1 \times(0$ to 6$)=\mathrm{C} 1$

- $\mathrm{B} 2 \mathrm{x}(0$ to 6$)=\mathrm{C} 2$

- B3 $x(0$ to 6$)=\mathrm{C} 3$

- B4 $x(0$ to 6$)=\mathrm{C} 4$

\section{Total score}

The PASI score is $\mathrm{C} 1+\mathrm{C} 2+\mathrm{C} 3+\mathrm{C} 4$.

The severity of lichen planus was evaluated by visual analogue scale (VAS). VAS is usually a horizontal line, in which the left end marked (0) as no pruritus or pain and the right end (10) marked as worst imaginable pruritus or pain, anchored by word descriptors at each end, as illustrated in Fig. (2). The patients mark on the line the point that they feel that it represents their perception of their current state ${ }^{(8)}$. 


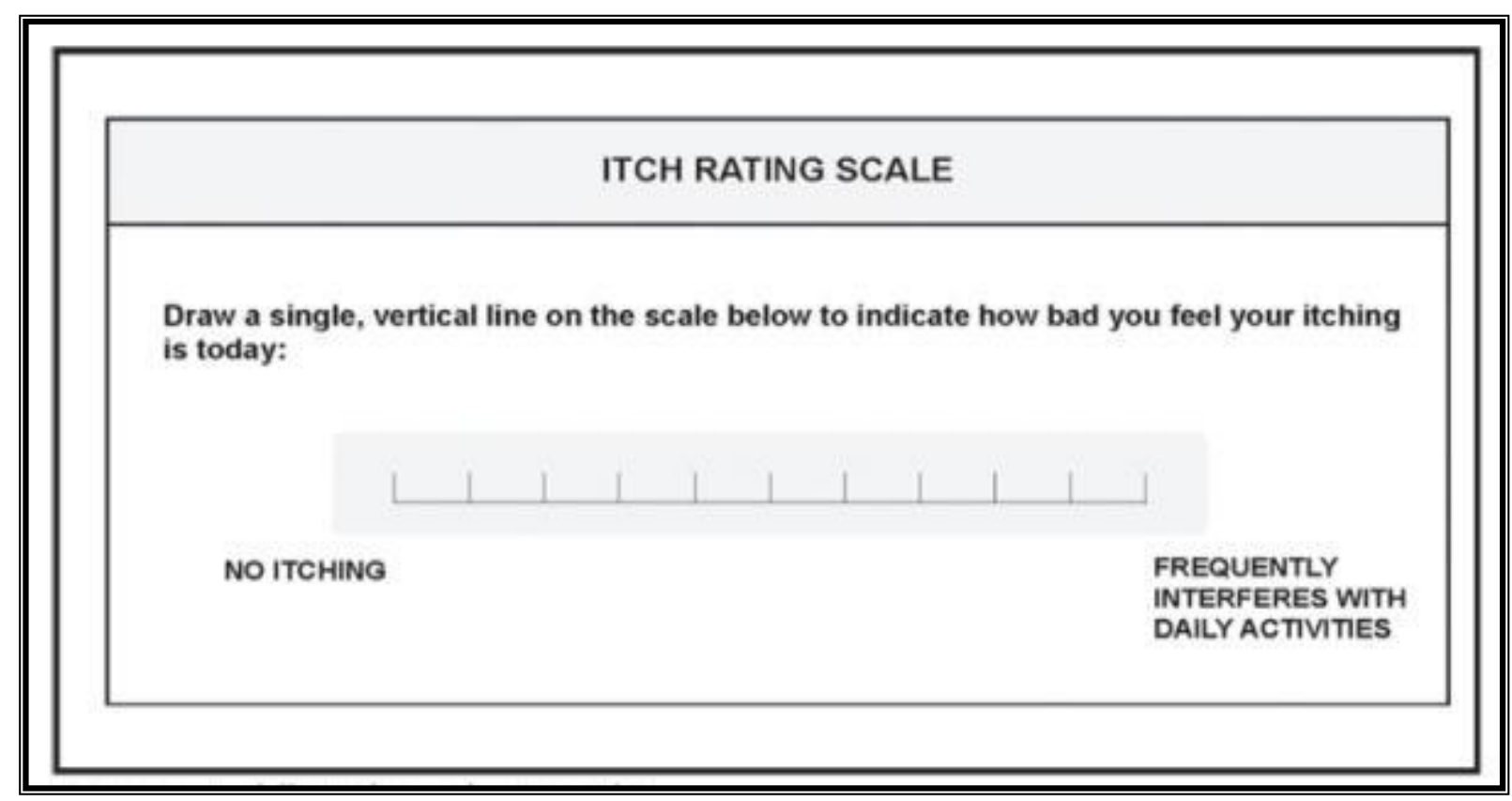

Fig. (2): Visual analog scale (VAS) ${ }^{(9)}$

\section{D- Determination of serum ANG 2 1. Sample collection}

- Three ml venous blood samples were obtained from each study subject by venous puncture to be collected in sterile plane tube and allowed to stand for 30 minutes at room temperature then centrifuged at $3000 \mathrm{rbm}$ for 10 minutes to separate the serum. Serum samples (The supernatant) were then removed using sterile pipette and stored frozen below $-80{ }^{\circ} \mathrm{C}$ until the time of analysis.

\section{Principle of Test:}

The kit uses a double-antibody sandwich enzyme-linked immunosorbent assay (ELISA) to assay the level of human angiopoietin 2 in samples. Angiopoietin 2 was added to monoclonal antibody enzyme, which was precoated with human angiopoietin 2 monoclonal antibody, incubation. Then, angiopoietin 2 antibodies labelled with biotin was added, and combined with streptavidin-HRP to form immune complex. Then incubation was carried out and washing again to remove the uncombined enzyme. Then chromogen solution A, B was added; the colour of the liquid changes into the blue, and with the effect of acid, the colour finally became yellow. The chroma of the colour and the concentration of the human substance Angiopoietin 2 of sample were positively correlated.

\section{Statistical Analysis}

Data were fed to the computer and analyzed using IBM SPSS software package version 20.0. (Armonk, NY: IBM Corp). Qualitative data were described using number and percent. The KolmogorovSmirnov test was used to verify the normality of distribution. Quantitative data were described using range (minimum and maximum), mean, standard deviation and median. Significance of the obtained results was judged at the 5\% level. The following teste were used: Chi-square test for categorical variables to compare between different groups. Fisher's Exact or Monte Carlo correction for chi-square when more than $20 \%$ of the cells have expected count less than 5 groups. F-test (ANOVA) for normally distributed quantitative variables to compare between more than two groups. Mann Whitney test for abnormally distributed quantitative variables to compare between two studied groups. Kruskal Wallis test for abnormally distributed quantitative variables to compare between more than two studied groups. Post Hoc (Dunn's multiple comparisons test) for pairwise comparisons. Wilcoxon signed ranks test for abnormally distributed quantitative variables to compare between two periods. Friedman test for abnormally distributed quantitative variables to compare between more than two periods or stages. Post Hoc Test (Dunn's) for pairwise comparisons. Spearman coefficient to correlate between two distributed abnormally quantitative variables.

\section{RESULTS}

The control group included 15 healthy controls, their ages ranged from 22-48 years with a mean of $38.53 \pm 8.43$, they were 9 male and 6 female. There were no statistically significant differences between the studied groups regarding age, sex, previous treatment and duration of the disease (Table 1). In this study, the severity of psoriasis ranged from mild $(66.7 \%)$, moderate $(20 \%)$ to sever $(13.3 \%)$ as shown in table (1). 
Regarding descriptive analysis of lichen cases, there were $46.7 \%$ of cases cutaneous lesions and only, $53.3 \%$ were in the form of combined oral and cutaneous lichen plans (Table 3 ).

The disease severity was evaluated by visual analogue scales (VAS). Their VAS ranged from 0 to 8 with an average of $4.20 \pm 2.62$ (Table 4).

The serum levels of angiopoietin 2 of the two diseases were significantly higher compared to the control group. The mean serum level of angiopoietin 2 of the control group was $0.14 \pm 0.02 \mathrm{ng} / \mathrm{ml}$, while it was $3.18 \pm 6.98 \mathrm{ng} / \mathrm{ml}$ in psoriasis and $2.61 \pm 4.26 \mathrm{ng} / \mathrm{ml}$ in lichen planus (Table 5). There was no statistically significant difference in serum angiopoietin 2 level as regards sex, age and treatment. However, there was statistically significant difference in serum angiopoietin 2 level as regards duration of lichen planus group (Table 6).

There was significant correlation between serum level of angiopoietin 2 and severity of psoriasis assessed by PASI, while there was no significant correlation between serum level of angiopoietin 2 and severity of lichen planus assessed by VAS (Table 7, 8).

The serum levels of angiopoietin 2 was significantly higher in patients with both cutaneous and oral LP compared to patients of cutaneous LP only (Table 9).

Table (1): Comparison between the three studied groups according to demographic data

\begin{tabular}{|c|c|c|c|c|c|c|c|c|}
\hline & \multicolumn{4}{|c|}{ Cases } & \multirow{2}{*}{\multicolumn{2}{|c|}{$\begin{array}{l}\text { Control } \\
(\mathbf{n}=15)\end{array}$}} & \multirow{3}{*}{ Test of Sig. } & \multirow{3}{*}{$\mathbf{P}$} \\
\hline & \multicolumn{2}{|c|}{$\begin{array}{c}\text { Psoriasis } \\
(n=15)\end{array}$} & \multicolumn{2}{|c|}{$\begin{array}{c}\text { Lichen } \\
(n=15)\end{array}$} & & & & \\
\hline & No. & $\%$ & No. & $\%$ & No. & $\%$ & & \\
\hline \multicolumn{9}{|l|}{ Age (years) } \\
\hline Min. - Max. & \multicolumn{2}{|c|}{$25.0-52.0$} & \multicolumn{2}{|c|}{$28.0-59.0$} & \multicolumn{2}{|c|}{$22.0-48.00$} & \multirow{3}{*}{$\begin{array}{c}F= \\
2.776\end{array}$} & \multirow{3}{*}{0.074} \\
\hline Mean \pm SD. & \multicolumn{2}{|c|}{$41.07 \pm 8.24$} & \multicolumn{2}{|c|}{$45.80 \pm 9.03$} & \multicolumn{2}{|c|}{$38.53 \pm 8.43$} & & \\
\hline $\begin{array}{l}\text { Median } \\
\text { (IQR) }\end{array}$ & \multicolumn{2}{|c|}{$\begin{array}{c}40.0 \\
(37.0-50.0)\end{array}$} & \multicolumn{2}{|c|}{$\begin{array}{c}47.0 \\
(40.0-52.0)\end{array}$} & \multicolumn{2}{|c|}{$\begin{array}{c}40.0 \\
(32.5-45.0)\end{array}$} & & \\
\hline \multicolumn{9}{|l|}{ Sex } \\
\hline Male & 11 & 73.3 & 9 & 60.0 & 9 & 60.0 & \multirow{2}{*}{$\begin{array}{c}\chi^{2}= \\
0.776\end{array}$} & \multirow{2}{*}{0.678} \\
\hline Female & 4 & 26.7 & 6 & 40.0 & 6 & 40.0 & & \\
\hline \multicolumn{9}{|l|}{ Treatment } \\
\hline No & 5 & 33.3 & 7 & 46.7 & - & - & \multirow{2}{*}{$\begin{array}{c}\chi^{2}= \\
0.556\end{array}$} & \multirow{2}{*}{0.456} \\
\hline Yes & 10 & 66.7 & 8 & 53.3 & - & - & & \\
\hline \multicolumn{9}{|c|}{ Duration of the disease (month) } \\
\hline Min. - Max. & \multicolumn{2}{|c|}{$1.0-72.0$} & \multicolumn{2}{|c|}{$1.0-60.0$} & \multicolumn{2}{|c|}{-} & \multirow{3}{*}{$\mathrm{U}=104.0$} & \multirow{3}{*}{0.723} \\
\hline Mean \pm SD & \multicolumn{2}{|c|}{$20.47 \pm 23.75$} & \multicolumn{2}{|c|}{$14.93 \pm 17.67$} & \multicolumn{2}{|c|}{-} & & \\
\hline $\begin{array}{l}\text { Median } \\
\text { (IQR) }\end{array}$ & \multicolumn{2}{|c|}{$\begin{array}{c}12.0 \\
(2.50-36.0)\end{array}$} & \multicolumn{2}{|c|}{$\begin{array}{c}8.0 \\
(4.5-18.0)\end{array}$} & \multicolumn{2}{|c|}{-} & & \\
\hline
\end{tabular}

$\chi^{2}$ : Chi square test F: F for ANOVA test U: Mann Whitney test $\mathrm{p}: \mathrm{p}$ value for comparing between the studied groups

Table (2): Descriptive analysis according to severity and PASI score in psoriasis cases $(\mathrm{n}=15)$

\begin{tabular}{|c|c|c|}
\hline & No. & $\%$ \\
\hline \multicolumn{3}{|l|}{ Severity } \\
\hline Mild & 10 & 66.7 \\
\hline Moderate & 3 & 20.0 \\
\hline Severe & 2 & 13.3 \\
\hline \multicolumn{3}{|l|}{ PASI score } \\
\hline Min. - Max. & \multicolumn{2}{|c|}{$4.20-27.90$} \\
\hline Mean \pm SD & \multicolumn{2}{|c|}{$10.77 \pm 6.96$} \\
\hline Median (IQR) & \multicolumn{2}{|c|}{$8.0(6.4-13.05)$} \\
\hline
\end{tabular}

Table (3): Descriptive analysis according to type of Lichen cases $(\mathrm{n}=15)$

\begin{tabular}{|c|c|c|}
\hline & No. & $\%$ \\
\hline \multicolumn{3}{|l|}{ Type of lichen } \\
\hline Cutaneous only & 7 & 46.7 \\
\hline Combined oral and cutaneous & 8 & 53.3 \\
\hline
\end{tabular}


Table (4): Descriptive analysis according to severity and VAS score in lichen cases $(\mathrm{n}=15)$

\begin{tabular}{|c|c|c|}
\hline & No. & $\%$ \\
\hline \multicolumn{3}{|l|}{ Severity } \\
\hline Mild & 7 & 46.7 \\
\hline Moderate & 5 & 33.3 \\
\hline Severe & 3 & 20.0 \\
\hline \multicolumn{3}{|l|}{ VAS score } \\
\hline Min. - Max. & \multicolumn{2}{|c|}{$0.0-8.0$} \\
\hline Mean \pm SD & \multicolumn{2}{|c|}{$4.20 \pm 2.62$} \\
\hline Median (IQR) & \multicolumn{2}{|c|}{4.0} \\
\hline
\end{tabular}

Table (5): Comparison between the three studied groups according to angiopoietin (ng/ml)

\begin{tabular}{|c|c|c|c|c|c|c|}
\hline \multirow{2}{*}{$\begin{array}{l}\text { Angiopoietin } \\
(\mathrm{ng} / \mathrm{ml})\end{array}$} & \multicolumn{2}{|c|}{ Cases } & \multirow{2}{*}{$\begin{array}{l}\text { Control } \\
(\mathbf{n}=15)\end{array}$} & \multirow[b]{2}{*}{$\mathbf{H}$} & \multirow[b]{2}{*}{$\mathbf{P}$} & \multirow{2}{*}{$\begin{array}{l}\text { Pairwise } \\
\text { comparison } \\
\text { P-value }\end{array}$} \\
\hline & $\begin{array}{c}\text { Psoriasis } \\
(\mathbf{n}=15)\end{array}$ & $\begin{array}{c}\text { Lichen } \\
(n=15)\end{array}$ & & & & \\
\hline Min. - Max. & $0.11-22.57$ & $0.15-12.36$ & $0.11-0.17$ & \multirow{3}{*}{$20.186^{*}$} & \multirow{3}{*}{$<0.001^{*}$} & \multirow{3}{*}{$\begin{array}{c}0.749^{* p 1} \\
<0.0011^{*}{ }^{2} \\
<0.0011^{*}{ }^{3}\end{array}$} \\
\hline Mean \pm SD & $3.18 \pm 6.98$ & $2.61 \pm 4.26$ & $0.14 \pm 0.02$ & & & \\
\hline $\begin{array}{l}\text { Median } \\
\text { (IQR) }\end{array}$ & $\begin{array}{c}0.24 \\
(0.19-0.78) \\
\end{array}$ & $\begin{array}{c}0.21 \\
(0.17-2.88) \\
\end{array}$ & $\begin{array}{c}0.15 \\
(0.13-0.16) \\
\end{array}$ & & & \\
\hline
\end{tabular}

$\mathrm{H}$ : $\mathrm{H}$ for Kruskal Wallis test, Pairwise comparison bet. each 2 groups was done using Post Hoc Test (Dunn's for multiple comparisons test) $\mathrm{p}$ : $\mathrm{p}$ value for comparing between the studied groups $\mathrm{p}_{1}$ : $\mathrm{p}$ value for comparing between Psoriasis and Lichen $\quad p_{2}: p$ value for comparing between Psoriasis and control

$\mathrm{p}_{3}: \mathrm{p}$ value for comparing between Lichen and control $\quad *$ : Statistically significant at $\mathrm{p} \leq 0.05$

Table (6): Correlation between angiopoietin $2(\mathrm{ng} / \mathrm{ml})$ with different parameters in each group

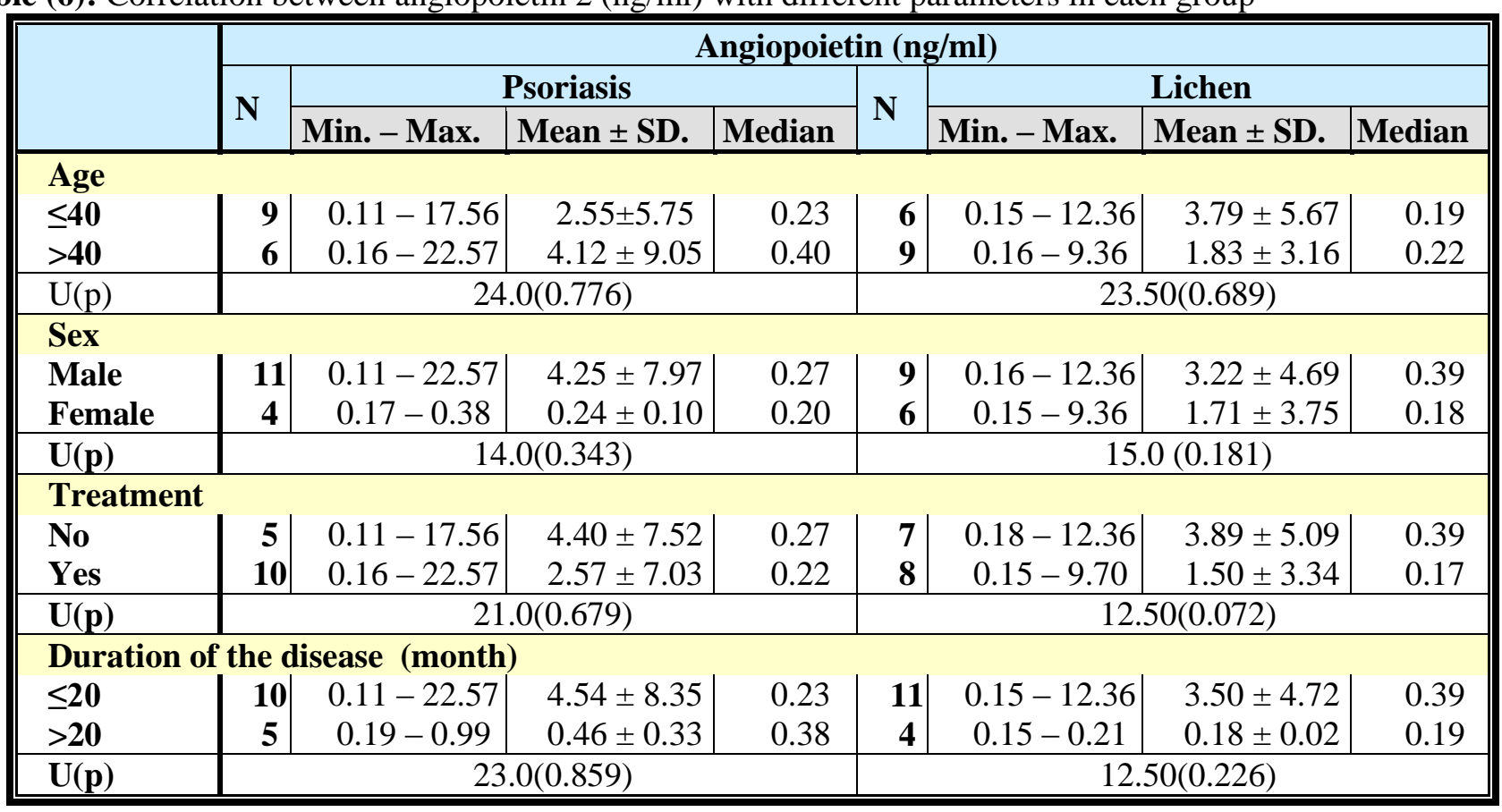

U: Mann Whitney test

$\mathrm{p}$ : $\mathrm{p}$ value for association between angiopoietin $2(\mathrm{ng} / \mathrm{ml})$ and different parameters

Table (7): Correlation between angiopoietin $2(\mathrm{ng} / \mathrm{ml})$ with disease severity in each group

\begin{tabular}{|l|c|c|c|c|}
\hline \multirow{2}{*}{} & \multicolumn{4}{|c|}{ Angiopoietin (ng/ml) } \\
\cline { 2 - 5 } & \multicolumn{2}{|c|}{ Psoriasis } & \multicolumn{2}{c|}{ Lichen } \\
\cline { 2 - 5 } & $\mathbf{r}_{\mathbf{s}}$ & $\mathbf{P}$ & $\mathbf{r}_{\mathbf{s}}$ & $\mathbf{P}$ \\
\hline PASI score & 0.596 & $0.019^{*}$ & - & - \\
\hline VAS score & - & - & 0.508 & 0.053 \\
\hline
\end{tabular}

$\mathrm{r}_{\mathrm{s}}$ : Spearman coefficient $\quad *$ : Statistically significant at $\mathrm{p} \leq 0.05$ 
Table (8): Relation between angiopoietin $2(\mathrm{ng} / \mathrm{ml})$ and severity in in each group

\begin{tabular}{|c|c|c|c|c|c|c|c|c|}
\hline \multirow{3}{*}{ Severity } & \multicolumn{8}{|c|}{ Angiopoietin (ng/ml) } \\
\hline & \multirow{2}{*}{$\mathbf{N}$} & \multicolumn{3}{|c|}{ Psoriasis } & \multirow[b]{2}{*}{$\mathbf{N}$} & \multicolumn{3}{|c|}{ Lichen } \\
\hline & & Min. - Max. & Mean \pm SD & Median & & Min. - Max. & Mean \pm SD. & Median \\
\hline Mild & 10 & $0.11-0.57$ & $0.25 \pm 0.14$ & 0.20 & 7 & $0.15-1.25$ & $0.33 \pm 0.41$ & 0.18 \\
\hline Moderate & 3 & $0.24-3.85$ & $1.69 \pm 1.91$ & 0.99 & 5 & $0.16-9.70$ & $4.78 \pm 4.68$ & 4.51 \\
\hline Severe & 2 & $17.56-22.57$ & $20.07 \pm 3.54$ & 20.07 & 3 & $0.21-12.36$ & $4.32 \pm 6.96$ & 0.39 \\
\hline $\mathrm{H}(\mathrm{p})$ & \multicolumn{4}{|c|}{$7.995^{*}\left(0.018^{*}\right)$} & \multicolumn{4}{|c|}{$3.864(0.145)$} \\
\hline
\end{tabular}

$\mathrm{H}$ : $\mathrm{H}$ for Kruskal Wallis test $\mathrm{p}$ : $\mathrm{p}$ value for association between angiopoietin $2(\mathrm{ng} / \mathrm{ml})$ and severity

*: Statistically significant at $\mathrm{p} \leq 0.05$

Table (9): Relation between Angiopoietin (ng/ml) and type of lichen in Lichen cases ( $\mathrm{n}=15)$

\begin{tabular}{|c|c|c|c|c|c|c|}
\hline \multirow{2}{*}{ Type of lichen } & \multirow{2}{*}{$\mathbf{N}$} & \multicolumn{3}{|c|}{ Angiopoietin (ng/ml) } & \multirow{2}{*}{$\mathbf{U}$} & \multirow{2}{*}{$\mathbf{p}$} \\
\hline & & Min. - Max. & Mean \pm SD. & Median & & \\
\hline Cautenous only & 7 & $0.15-0.22$ & $0.18 \pm 0.02$ & 0.18 & \multirow{2}{*}{$11.0^{*}$} & \multirow{2}{*}{$0.049^{*}$} \\
\hline Combined oral and cautenous & 8 & $0.15-12.36$ & $4.74 \pm 5.03$ & 2.88 & & \\
\hline
\end{tabular}

U: Mann Whitney test p: p value for association between angiopoietin 2 (ng/ml) and type of lichen

*: Statistically significant at $\mathrm{p} \leq 0.05$

\section{DISCUSSION}

As regards psoriasis in the current study, there were significantly high serum levels of angiopoietin $2(\mathrm{p}<0.001)$ in psoriasis patients (median $=0.24)$ in comparison with controls (median $=0.15$ ). A study has identified high levels of ang-2 expression in serum (median $=1.01)$ that was positively correlated with disease severity measured by PASI score (10). While He $\boldsymbol{e t}$ al. ${ }^{(11)}$ assessed angiopoietin 2 in angiogenic genes and found that Ang-2 is induced in papillary dermis of psoriatic skin and elucidated that genetic variations in the Ang-2 gene may therefore affect vascular development and angiogenesis in psoriasis.

In current study, $66.7 \%$ of psoriasis patients received treatment but $33.3 \%$ did not receive any treatment, with non-significant difference $(\mathrm{p}=$ 0.456). While, with Takahashi et al. ${ }^{(10)}$, all patients had been on topical steroid and four patients underwent antitumor necrosis factor therapy and that was significant $(p=0.083)$. So, the evaluation of therapeutic influence on serum Ang 2 levels revealed that anti TNF therapy suppressed their levels in all patients as it is speculated that TNF-a induces release of Ang 2 from Weibel-Palade bodies and promotes adhesion of inflammatory cells to endothelial cells leading to initiation of psoriatic inflammation as well as activation of angiogenesis.

In the current study, we found that serum Ang-2 levels were significantly higher in PsV patients than in healthy controls $0.24(0.19-0.78)$ vs $0.15(0.13-0.16, \mathrm{P}=0.001)$. This is in accordance with Takahashi et al. ${ }^{\left({ }^{(10)}\right.}$ who found that serum Ang 2 levels were significantly higher in $\mathrm{PsV}$ patients than in healthy controls [1.12-2.06] vs $1.01 \mathrm{ng} / \mathrm{mL}$ [0.84-1.32], $(\mathrm{P}=0.032)$. This indicates that dysregulation of Ang 2 contributes to the development of both local skin lesions and the systemic events of psoriasis in addition to that; it suggests the potential usefulness of serum Ang 2 level as a biomarker of PsV.

We next examined the correlation of serum Ang-2 levels with PASI score, and found a significant correlation between these two factors ( $p$ $=0.019)$. This finding is also in concordance with those of another study conducted on psoriasis patients by Takahashi et al. ${ }^{(10)}$ who reported that there was a significant correlation between serum Ang 2 and PASI score $(\mathrm{p}=0.011)$. In other words, a higher disease extent might account for a higher change in Ang 2 as an important mediator in disease pathogenesis and severity.

Evidence that supports the more important role of Ang-2 in the pathogenesis of psoriasis is shown by Kurouda et al. ${ }^{(\mathbf{1 2})}$, who elucidated the potential role of the angiopoietin 2 in vascular changes of psoriasis by examining the expression and localization of Ang-2 in psoriasis skin and histopathologic studying of the cytokine-mediated regulation of this molecule in cell cultures. This study revealed that Ang-2 was present in the papillary dermis of involved psoriasis skin and suggested that upregulation of angiopoietin 2 is closely associated with the development of vascular expansion and promotion of neovascularization in psoriasis. Moreover, this study showed an association between improvement in skin lesions because of antipsoriatic therapy and decreased levels of angiopoietin and Tie 2 in skin lesions. In particular, successful antipsoriatic treatment dramatically reduced Ang-2 expression in involved skin to levels of uninvolved skin, and this reduction appears to occur early during therapy. These observations suggest that alteration of Ang-2 
expression levels may be particularly important in controlling vascular proliferation in psoriasis.

Concerning lichen planus, we found that serum Ang-2 levels were statistically significant higher in lichen planus patients than in healthy controls $(0.21(0.17-2.88)$ vs $0.15(0.13-0.16 \mathrm{P}=$ $0.001)$.

We next examined the correlation between serum Ang-2 levels and the presence of oral lesions plus the cutaneous ones. We found that the levels to be higher in the presence of oral lesions and was statistically significant $(\mathrm{p}=0.049)$.

Few studies have investigated angiogenesis in LP, such as Desanka et al. ${ }^{(13)}$ who highlighted a potent angiogenic activity in the CLP lesions and found that the blood microcirculatory bed in the CLP lesions was approximately 1.6 times more extensive than in the control healthy skin. Furthermore, provided novel morphological evidence of a potent lymphangiogenic activity in the CLP lesions compared to the healthy skin. The lymphatic microcirculation in the CLP lesions was approximately 1.8 times more extensive. Similarly, Al hassiny et al. (14) whose data suggested that angiogenesis also plays an important role in OLP lesions and that angiogenesis may be an underlying marker of OLP disease activity, and showed that vascular adhesion molecules, which allow interaction between EC and lymphocytes have been shown to be expressed significantly more in OLP by comparison with normal oral mucosa.

\section{CONCLUSION}

The current study provided an important evidence on the importance of angiopoietin 2 level in pathogenesis of lichen planus as well as psoriasis as it elevated in serum of psoriasis and lichen planus patients that reflects its role in angiogenesis and inflammation, which are important events in the development of these diseases.

Given the active role of Ang-2 in these diseases, targeting the Ang/Tie pathway is a promising approach, especially now. The current anti-angiogenic therapies are under serious consideration, and the existing clinical data is encouraging. Therefore, more research on large number of patients is needed to confirm the results and further studies are required to understand the contradicting roles of Ang-2 and the Ang-2 induced signaling circuitries in psoriasis and lichen planus diseases. In addition, to compare angiopoietin 2 level before and after treatment by different treatment modalities with the ultimate goal of developing potential therapeutic targets.

\section{REFERENCES}

1. Nograles K, Davidovici B, James G (2011): IL-23/TH17 Pathway in Psoriasis and Inflammatory Skin Diseases. S. Jiang (ed.), T H17 Cells in Health and Disease. https://www.scireslit.com/Dermatology/SJCRDID26.pdf

2. Li X, Li J, Yang Y et al. (2013): Differential gene expression in peripheral blood $\mathrm{T}$ cells from patients with psoriasis, lichen planus, and atopic dermatitis. J Am Acad Dermatol., 65: 6-30

3. Norooznezhad A, Fatemeh $\mathbf{N}$ (2017): Cannabinoids: Possible agents for treatment of psoriasis via suppression of angiogenesis and inflammation. Medical Hypotheses, 99: 15-18.

4. Shibuya M (2013): Vascular endothelial growth factor and its receptor system: physiological functions in angiogenesis and pathological roles in various diseases. The Journal of Biochemistry, 153 (1): 13-19.

5. Fagiani E, Christofori G (2013): Angiopoietins in angiogenesis. Cancer Lett., 328: 18-26.

6. Scholz A, Plate K, Reiss Y (2015): Angiopoietin-2: a multifaceted cytokine that functions in both angiogenesis and inflammation. Annals of the New York Academy of Sciences, 1347: 45-51.

7. Robinson A (2012): Psoriasis severity scoring. http://www. dermnetnz.org/scaly/pasi.html

8. Wu C, Wu C (2008): Life satisfaction in persons with schizophrenia living in the community validation of the satisfaction with life scale. Social Indicators Research, 85: 447-460.

9. Kircik H (2011): Efficacy and onset of action of hydrocortisone acetate $2.5 \%$ and pramoxine hydrochloride $1 \%$ lotion for the management of pruritus: results of a pilot study. The Journal of Clinical and Aesthetic Dermatology, 4 (2): 48-55.

10. Takahashi T, Asano Y, Shibata S et al. (2017): Serum angiopoietin-2 level as a potential biomarker in psoriasis vulgaris. The Journal of Dermatology, 44 (2): 205-206.

11. He L, Dang L, Zhou J et al. (2015): Association of angiopoietin-1, angiopoietin-2 and caspase-5 polymorphisms with psoriasis vulgaris. Clinical and Experimental Dermatology, 40 (5): 556-563.

12. Kuroda K, Sapadin A, Shoji T et al. (2001): Altered expression of angiopoietins and Tie2 endothelium receptor in psoriasis. J Invest Dermatol., 116: 713-20.

13. Desanka V, Mellová $Y$, Adamicová $K$ et al. (2015): Quantitative immunohistochemical assessment of blood and lymphatic microcirculation in cutaneous lichen planus lesions. Histol Histopathol., 30 (6): 697-706.

14. Al-Hassiny A, Friedlander L, Parachuru $\mathrm{V}$ et al. (2018): Upregulation of angiogenesis in oral lichen planus. Journal of Oral Pathology \& Medicine, 47 (2): 173178. 